\title{
Improved Error Correction Methods for Filterless Digital Class D Audio Power Amplifier Based on FCLNF
}

\author{
Li Li $\mathbb{D}^{1,2}$ Hong-jie Li, ${ }^{2}$ and Yan-jing Sun ${ }^{1}$ \\ ${ }^{1}$ School of Information and Control Engineering, China University of Mining and Technology, Xuzhou 221116, China \\ ${ }^{2}$ Department of Electronic Information and Electrical Engineering, Anyang Institute of Technology, Anyang 455000, China
}

Correspondence should be addressed to Li Li; lilifkb@163.com

Received 4 May 2020; Revised 19 July 2020; Accepted 22 July 2020; Published 12 August 2020

Guest Editor: Sanghyuk Lee

Copyright (c) $2020 \mathrm{Li} \mathrm{Li}$ et al. This is an open access article distributed under the Creative Commons Attribution License, which permits unrestricted use, distribution, and reproduction in any medium, provided the original work is properly cited.

\begin{abstract}
Aiming at correcting the error caused by the nonlinear and power supply noise of the bridge-tied-load (BTL) power stage of the filterless digital class D power amplifier, an error correction method was proposed based on feedforward power supply noise suppression (FFPSNS) and first-order closed loop negative feedback (FCLNF) techniques. This method constructed the first-order LCLNF loop for the power stage and further reduced the impact of the power supply noise on the power amplifier output by using FFPSNS technology to introduce the power supply noise into the feedback loop at the same time. The $0.35 \mu \mathrm{m}$ CMOS process is used for analysis and comparison in Cadence. Cadence simulation results indicate that PSRR at the power supply noise frequency of $200 \mathrm{~Hz}$ is improved with $36.02 \mathrm{~dB}$. The power supply induced intermodulation distortion (PS-IMD) components are decreased by approximately $15.57 \mathrm{~dB}$ and the signal-to-noise ratio (SNR) of the power amplifier is increased by $17 \mathrm{~dB}$. The total harmonic distortion + noise $(\mathrm{THD}+N)$ of the power amplifier is reduced to $0.02 \%$ by FCLNF + FFPSNS.
\end{abstract}

\section{Introduction}

In recent years, the country has promoted high-efficiency and energy-saving technologies and encouraged the strengthening of high-efficiency and energy-saving technological transformations and key technological breakthroughs. With the rise of audio and video equipment for low-power applications, filter-free digital class D audio power amplifiers have high power efficiency and easy interface with digital audio sources, which are favored by researchers in the industry [1]. And in the 11 key technologies evaluated by IEEE Spectrum in the past ten years, it is predicted that high-efficiency class D audio amplifiers will eventually unify the audio amplifier market [2]. However, its own nonideal state and power supply noise can cause serious distortion of the output signal of the power amplifier.

Digital class $\mathrm{D}$ power amplifiers have received increasing attention due to their advantages of high power efficiency, easy system transplantation, and resistance to external interference [3]. The traditional digital class $\mathrm{D}$ power amplifier is usually composed of a digital switching signal modulator, a power stage, and an inductive capacitor (LC) analog lowpass filter. The digital switching signal modulator mostly uses uniform-sampling pulse width modulation (UPWM) technology realization. The LC low-pass filter of the traditional digital class D power amplifier occupies about $75 \%$ of the volume of the entire power amplifier system and consumes about $30 \%$ of the cost, which severely hinders the portable application of digital class D power amplifiers [4]. The filter-free digital class D power amplifier, as a new type of digital class $\mathrm{D}$ power amplifier, can drive the loudspeaker to work without the need of an LC low-pass filter through a special modulation technique and maintain high power efficiency to meet the needs of digital audio with the development of equipment miniaturization; the filter-free digital class D power amplifier has become a research hotspot in the current power amplifier field [5].

In order to enable the digital class $\mathrm{D}$ audio power amplifier to achieve high power supply rejection ratio (PSRR), power supply-induced intermodulation Distortion (PS-IMD), signal-to-noise rate (SNR), and total harmonic distortion + noise $(\mathrm{THD}+N)$, two large error sources need 
to be corrected for the nonidealities and the power level source noise introduced by the power level [6].

For filter-free digital class D power amplifiers, the errors introduced by the nonlinearity and nonideal working conditions of the power stage, especially the errors introduced by the power supply noise of the power stage, seriously affect the output performance of the power amplifier $[7,8]$. Although the power amplifier's power supply rejection ratio (PSRR) can theoretically reach infinity when the upper and lower half bridges of the bridge-tied-load (BTL) power stage of the power amplifier are completely matched, the power supply interference is caused by the power supply noise. The modulation distortion (PS-IMD) is still large. In response to this problem, Donida et al. [7] and Mostert et al. [8] introduced the output signal of the power amplifier to the input of the power amplifier by establishing a global closedloop negative feedback loop including a digital switching signal modulator and a power stage. Precorrection is performed in the terminal or switching signal modulator to achieve the purpose of correcting the power level error, but an additional analog-to-digital converter (ADC) is required, which leads to a substantial increase in system cost. Chen et al. [9] and Cellier et al. [10] built a local closed loop negative feedback (LCLNF) loop containing only the power level to correct the power level error. This method is ensuring the system. In a stable condition, the ability to suppress the noise of the power level power supply is weak. In summary, there are currently fewer error correction methods for the power stage of the filter-free digital class D power amplifier, and the existing digital class $\mathrm{D}$ power amplifier power stage error correction method may have a higher cost of implementation, or the power stage power error. The correction ability is weak.

Aiming at the above two major error sources, Dong Jun Lee and Jinho Noh et al. used the local negative feedback technology to establish a power-level noise suppression module between the switch signal modulator and the power level, but it would cause an increase in the power level switching frequency $[11,12]$. There are also the use of analog negative feedback control loop schemes and the use of power-level power supply noise feedforward precorrection technology [13-15]. The scheme is complex and requires the use of $\mathrm{ADC}$ to convert power source noise into digital signals. In [16], a feedforward noise technology for class D audio amplifier with single end (SE) output structure is proposed, but this method is applied to class D audio amplifier with SE output structure, and the system contains an off-chip LC low-pass filter. This paper designs a first-order closed-loop negative feedback (FCLNF) error correction method for the filter-free digital class $\mathrm{D}$ audio power amplifier bridge-tied-load (BTL) power stage and the FCLNF suitable for the filter-free digital class $\mathrm{D}$ audio power amplifier BTL power stage plus feedforward power supply noise suppression (FCLNF + FFPSNS) error correction method.

\section{FCLNF + FFPSNS Error Correction}

Class D power stage is one of the key modules of the filterfree digital class D amplifier. Its function is to amplify the weak PWM signal to drive the low impedance speaker. Its input signal and output signal are both digital PWM signals. Since the ripple noise contained in the power supply and the nonideal state of the power stage itself affect the quality of the output signal, it is necessary to perform error correction on the open loop power stage.

Filter-free digital class D audio power amplifier is mainly composed of digital UPWM modulator and BTL power stage. The former is a digital part and the latter is an analog part. Its structure is shown in Figure 1. It can be seen from Figure 1 that the filter-free digital class $\mathrm{D}$ audio power amplifier can be divided into UPWM modulator, open-loop BTL power stage, and loudspeaker as a whole. The UPWM modulator is a digital circuit, and the open-loop BTL power stage is an analog circuit. UPWM modulator consists of the digital interpolation filter, in-phase and inverted unity gain buffer, Sigma-Delta modulator, and UPWM generator.

2.1. Principle Analysis of FCLNF Error Correction. FCLNF circuit is composed of compensation module, remodulation module, BTL power level, and some passive components $[3,17]$. Its circuit and equivalent model are shown in Figure 2. There $V_{\text {IN_P }}$ and $V_{\text {IN_N }}$ are, respectively, the input signals and $V_{\mathrm{CM}}$ is the common mode voltage or reference voltage.

$F_{1}$ and $F_{2}$ are transfer functions from the input signal of $V_{\text {IN_P }}$ and $V_{\text {IN_N }}$ to the inverting input of the integrator. $G_{\text {int } 1}$ and $G_{\text {int2 }}$ are the integrator gains of the upper and lower bridges, respectively. $H_{1}$ and $H_{2}$ are the feedback factors of the upper and lower bridges, respectively. $G_{M 1}$ and $G_{M 2}$ are the combined linear gain of the power level and the remodulation module.

Under ideal conditions,

$$
G_{M 1}=G_{M 2}, V_{\text {IN_P }}=V_{\text {IN_N }}=0,
$$

if the deviation between the two feedback paths is $\varepsilon$.

The mismatch factor between resistances is $r(0<r<1)$, mismatch factor between capacitors is $c(0<c<1)$, and $\varepsilon$ is equivalent to $r+c$,

The PSRR of power amplifier using the FCLNF method is

$$
\operatorname{PSRR}_{\mathrm{FCLNF}}=20 \lg \left[L G_{2}(s)\right]-20 \lg (r+c) .
$$

\subsection{Principle Analysis of FCLNF + FFPSNS Error Correction.} FFPSNS feeds the power stage power supply noise forward to the power stage front-end module for precorrection through certain means. The feedforward control network subtracts the feedforward power supply noise from the PWM input signal and then quantifies the power supply noise through the comparator. The power supply noise is converted into the effective duty cycle of PWM signal, so as to achieve the purpose of correcting the power supply noise. This proposal is composed of FCLNF module, FFPSNS module, remodulation module, and BTL power level $[18,19]$. Its schematic diagram and equivalent model are shown in Figure 3.

There $V_{\text {IN_P }}$ and $V_{\text {IN_N }}$ are, respectively, the input signals of the upper and lower half bridges of the power port in the power amplifier. $V_{\mathrm{CM}}$ is the common mode voltage or 


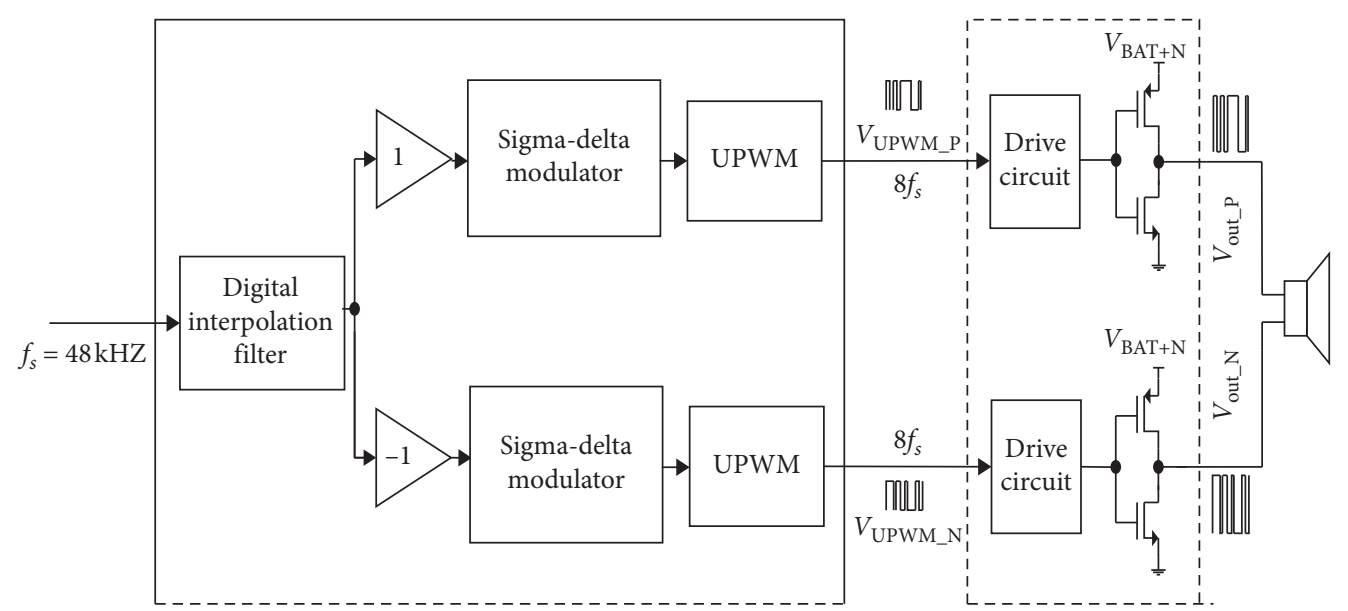

FIGURE 1: Block diagram of filterless digital class D power amplifier.

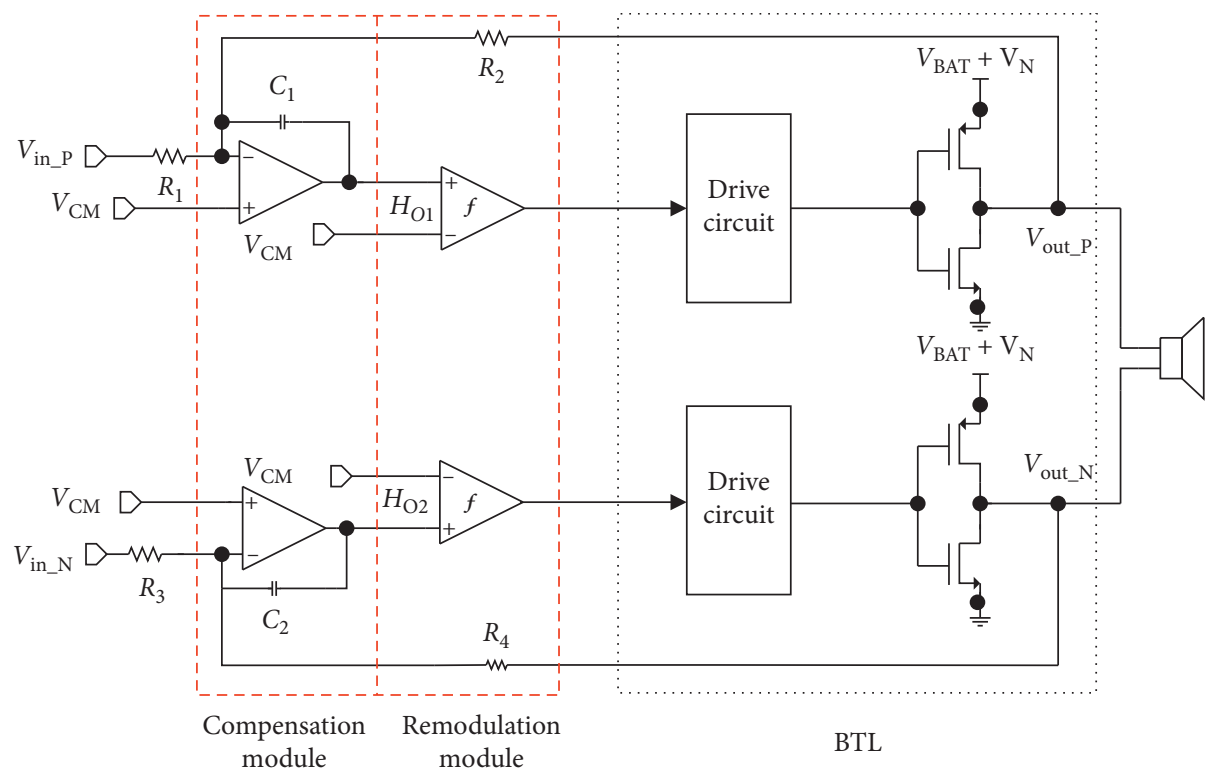

(a)

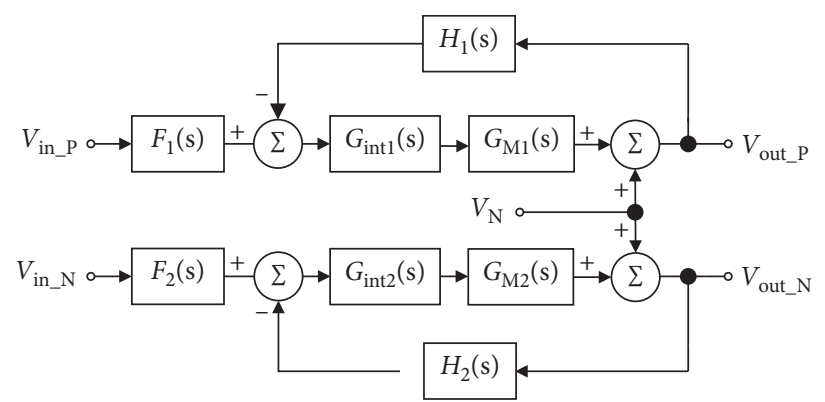

(b)

FIGURE 2: Diagram of FCLNF correction circuit. (a) Block diagram of FCLNF correction. (b) Mathematical model diagram of FCLNF correction.

reference voltage. $R_{f}$ is feedback resistance. $V_{A 1}$ and $V_{A 2}$ are the output signals of the integrator. $V_{F 1}$ and $V_{F 2}$ are the output signals of FFPSNS module. $R_{1}-R_{5}, R_{f}, R_{i}, C_{1}$ and $R_{1}^{\prime}-R_{5}^{\prime}, R_{f}^{\prime}, R_{i}^{\prime}, C_{1}^{\prime}$ have the same physical meaning and value.
$F_{1}$ and $F_{2}$ are transfer functions from the input signal of $V_{\text {IN_P }}$ and $V_{\text {IN_N }}$ to the inverting input of the integrator. $G_{\text {int } 1}$ and $G_{\text {int } 2}$ are the integrator gains of the upper and lower bridges, respectively. $H_{1}$ and $H_{2}$ are the feedback factors of the upper and lower bridges, respectively. $G_{M 1}$ and 


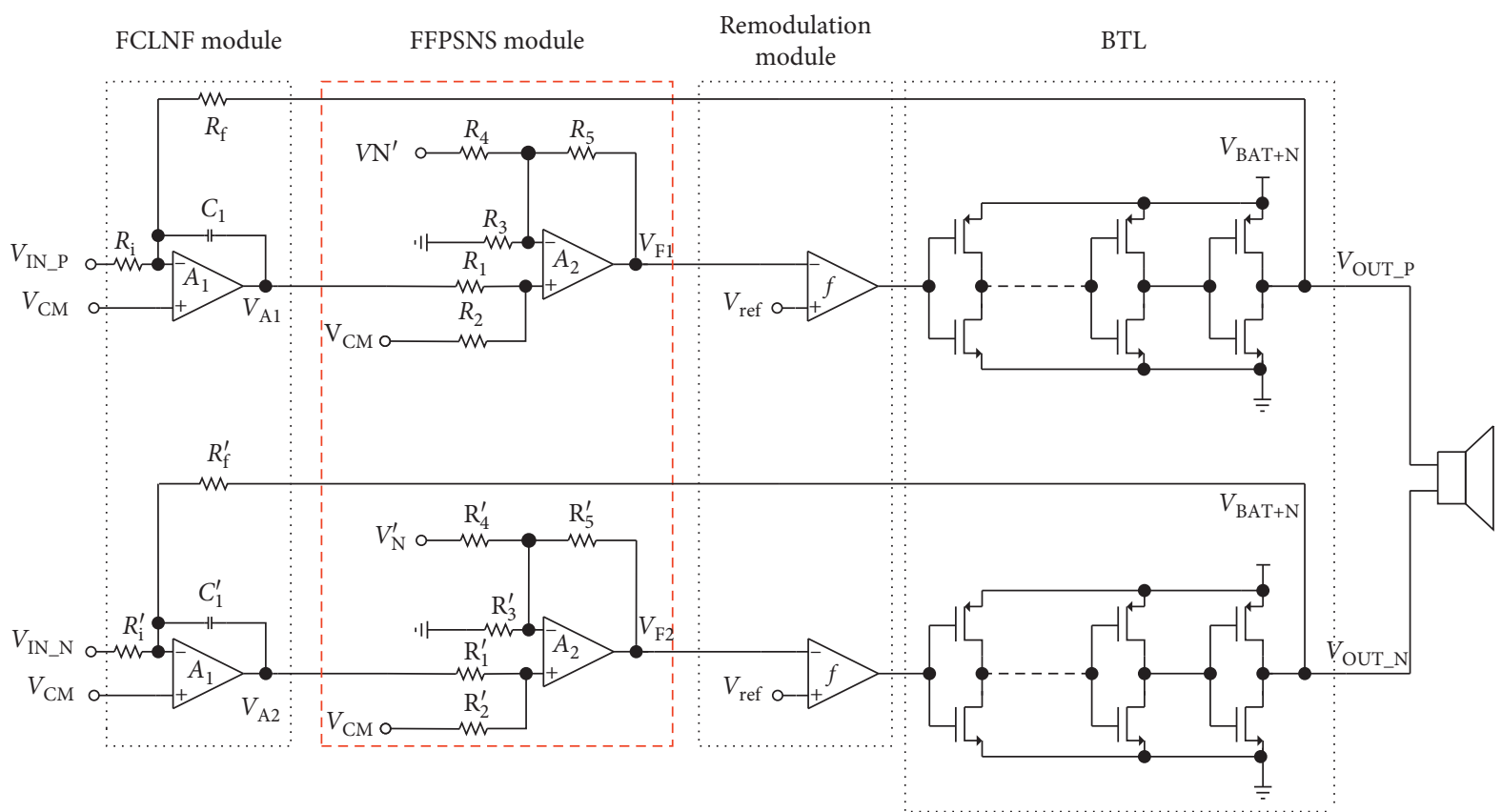

(a)

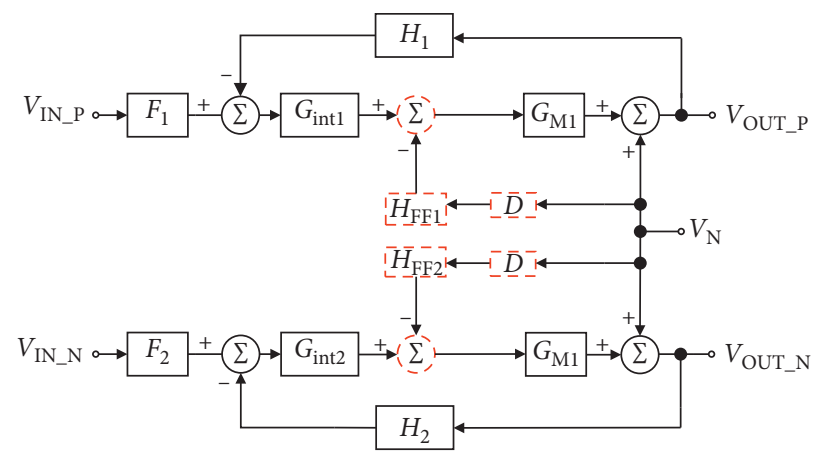

(b)

FigURE 3: Diagram of FCLNF + FFPSNS correction circuit. (a) Block diagram of FCLNF + FFPSNS correction. (b) Mathematical model diagram of FCLNF + FFPSNS correction.

$G_{M 2}$ are the combined linear gain of the power level and the remodulation module. $D$ is the power supply noise scaling factor. $H_{F F 1}$ and $H_{F F 2}$ are the transfer function of feedforward path.

Under ideal conditions, $H_{F F 1}=H_{F F 2}, G_{M 1}=G_{M 2}$, and $V_{\text {IN_P }}=V_{\text {IN_N }}=0$ if the deviation between the two feedback paths is $\varepsilon$.

The deviation between $H_{F F}$ theory and actual situation is $\beta$. The mismatch factor between resistances is $r(0<r<1)$, mismatch factor between capacitors is $r(0<c<1)$, and $\varepsilon$ is equivalent to $r+c$.

The PSRR of power amplifier using the FCLNF+ FFPSNS method is

$$
\begin{aligned}
& \text { PSRR }_{\mathrm{FCLNF}+\mathrm{FFPSNS}} \\
& \quad=20 \lg \left[L G_{2}(s)\right]-20 \lg (r+c)-20 \lg (|\beta|) .
\end{aligned}
$$

2.3. Circuit Design of Improved Error Correction Method. The double-input single-output folding cascode two-stage Miller compensation operational amplifier used in this paper is shown in Figure 4.

Based on the proposed power stage error correction method, the circuit implementation of the filter-free digital class D power amplifier BTL power stage with error correction is implemented. It can be seen from Figure 1 that the operational amplifier and voltage comparator are the key modules for this method. The operational amplifier $A_{1}$ uses a double-input single-output folding cascode two-stage Miller compensation architecture, as shown in Figure 4. In the figure, $V_{D D}$ is the power supply voltage; $V_{b 1}, V_{b 2}, V_{b 3}$, and $V_{b 4}$ are the bias voltages of the operational amplifier; $V_{O}$ is the output signal of the operational amplifier; $V_{\text {in } 1}$ and $V_{\text {in2 }}$ are the input signals of the operational amplifier; $C_{m}$ is the Miller compensation capacitor; and resistance is $R_{m}$. By 


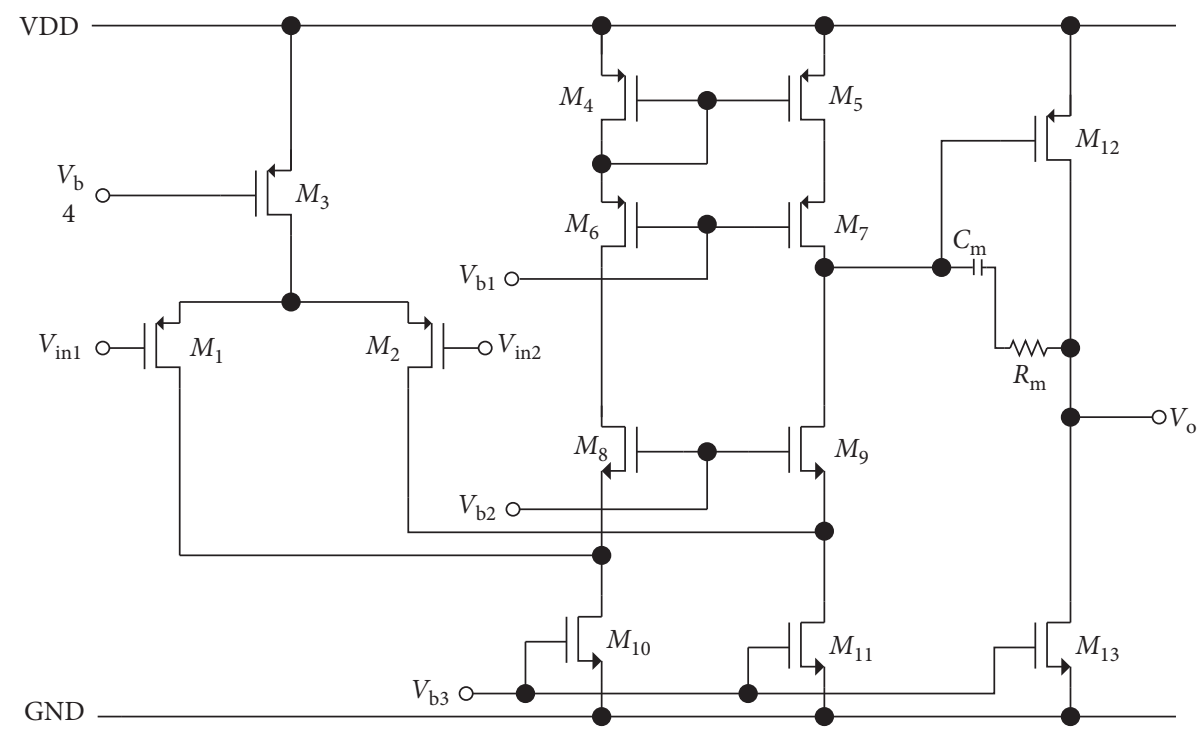

FIgURE 4: Structure of the operational amplifier.

connecting with $C_{m}$ in parallel to improve the stability of the operational amplifier, the unity gain bandwidth and phase margin of the operational amplifier $A_{1}$ are $13 \mathrm{MHz}$ and $70.3^{\circ}$, respectively, and the open loop gain is $109.9 \mathrm{~dB}$, and the PSRR at $100 \mathrm{~Hz}$ is $95 \mathrm{~dB}$. The feedforward operational amplifier $A_{2}$ also uses the architecture of Figure 4, with unity gain bandwidth and phase margin of $10 \mathrm{MHz}$ and $68^{\circ}$, respectively.

The voltage comparator circuit structure is relatively simple, and its circuit is shown in Figure 5. In the figure, $V_{\text {inl }}$ and $V_{\text {in } 2}$ are the input signals of the voltage comparator and $V_{O}$ is the output signal of the voltage comparator. The comparator uses a two-stage amplification architecture. The first stage of amplification uses a differential amplifier circuit structure and a differential circuit to suppress common mode interference. The second-stage amplification adopts a cascode circuit to control the offset voltage well. The output stage adopts the push-pull output to increase the driving capability of the output, while adding an inverter to the output stage can enhance the response time of the comparator. After simulation, the output waveform of the comparator has a rise time of $560 \mathrm{ps}$ and a fall time of $720 \mathrm{ps}$.

2.4. Simulation and Result Analysis. In order to verify the error correction effect of the above scheme, the simulation experiment route adopted in this paper is shown in Figure 6. The experimental simulation in this paper is based on Matlab and Cadence. Specifically, Matlab is used to generate the two PWM signal sources required by Cadence simulation, and the signal data exported by Matlab is saved as.dat file. In Cadence, $V_{\text {source }}$ module is used to store the waveform data in pwl type in Cadence and use it as the signal for Cadence simulation Source. FCLNF control circuit containing BTL power level was built on Cadence platform, and then Cadence Spectre simulator was used for transient simulation of the system. Finally after sampling in Cadence, import the.csv file into Matlab for spectrum analysis and performance calculation.

In this scheme, ASMC $0.35 \mu \mathrm{m}$ CMOS process is adopted to design and build the circuit on Cadence platform. The simulation conditions are mos_tt process angle and the temperature is $27^{\circ} \mathrm{C}$. FCLNF control circuit works under $5 \mathrm{~V}$ DC power supply voltage, that is, $V_{D D}$ is $5 \mathrm{~V}$, while the BTL power stage works under $10 \mathrm{~V} \mathrm{DC}$ power supply voltage. VCM is half of the working voltage of the negative feedback control circuit, and an $8 \Omega$ resistor is used as the load RL of the BTL power stage. The input signal of power amplifier is 24-bit sinusoidal single frequency digital signal (frequency is $1 \mathrm{kHz}$ and amplitude is $-5 \mathrm{~dB}$ ). The UPWM waveform data generated by Matlab is input into the simulation circuit through the $V_{\text {source }}$ component in Cadence. The transient simulation time is $42.7 \mathrm{~ms}$. The simulation output waveform is sampled and exported at an interval of $40.69 \mathrm{~ns}$ (the sampling frequency is $24.6 \mathrm{MHz}$ ). The simulation result is shown in Figure 7.

In Figure 7, after FCLNF correction, the power amplifier's PSRR at $200 \mathrm{~Hz}$ power source noise frequency was equal to $82.8 \mathrm{~dB}$, and the PSRR increased by $36.02 \mathrm{~dB}$. The PS-IMD of the power amplifier is approximately equal to $-80.63 \mathrm{~dB}$, reducing by $34.73 \mathrm{~dB}$. The SNR was increased to $85.7 \mathrm{~dB}$ and the SNR performance was much higher than that of the output signal of the uncorrected scheme $(58.59 \mathrm{~dB})$, which improved by $27.11 \mathrm{~dB}$. The output $\mathrm{THD}+N$ performance of the power amplifier was reduced to $0.0356 \%$, far less than the output THD $+N(0.464 \%)$ of the power amplifier without correction scheme. Figure shows that the PSRR of FCLNF + FFPSNS corrected power amplifier at $200 \mathrm{~Hz}$ power noise frequency is $84.3 \mathrm{~dB}$, which is close to the performance of PSRR in FCLNF.

The influence of power level source noise amplitude on the performance of power amplifier PSRR and PS-IMD is shown in Figures 8 and 9. 


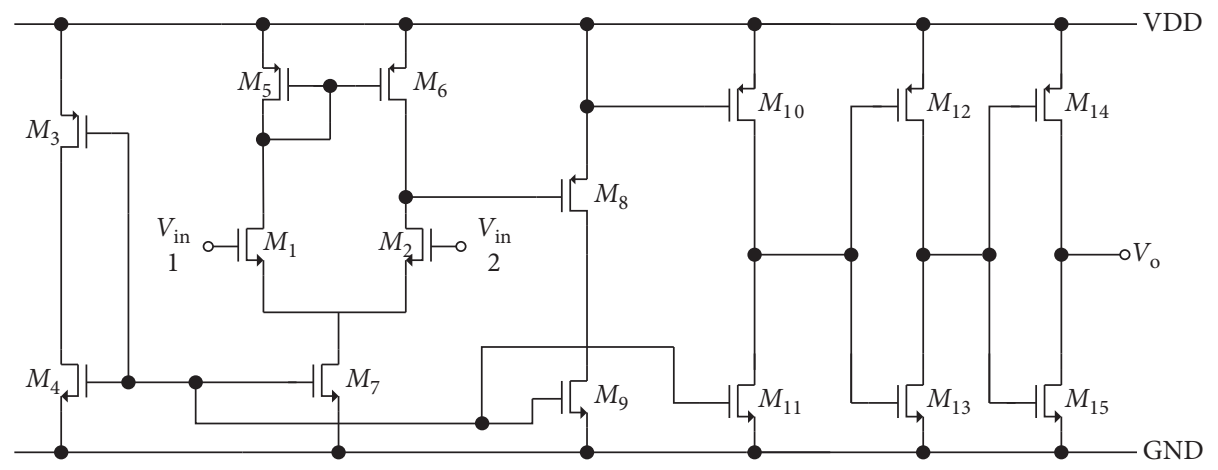

FIgURE 5: Structure of the voltage comparator.

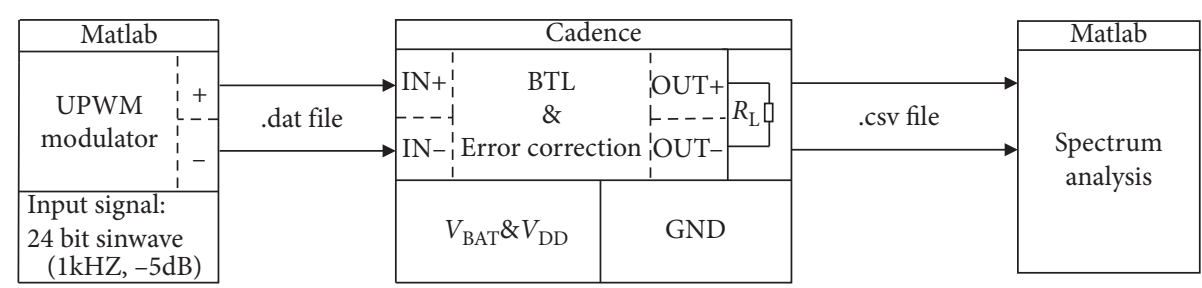

Figure 6: Block diagram of experimental.

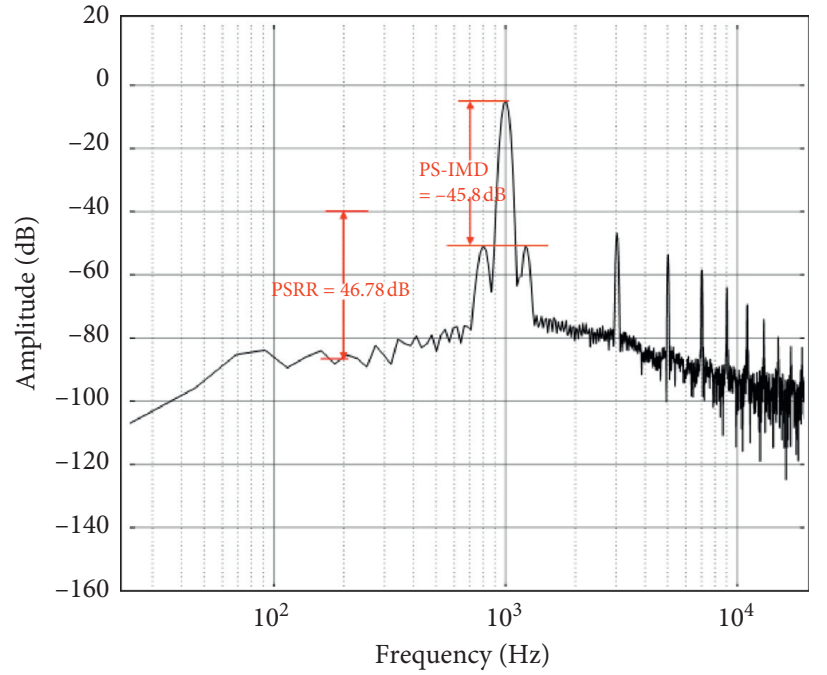

(a)

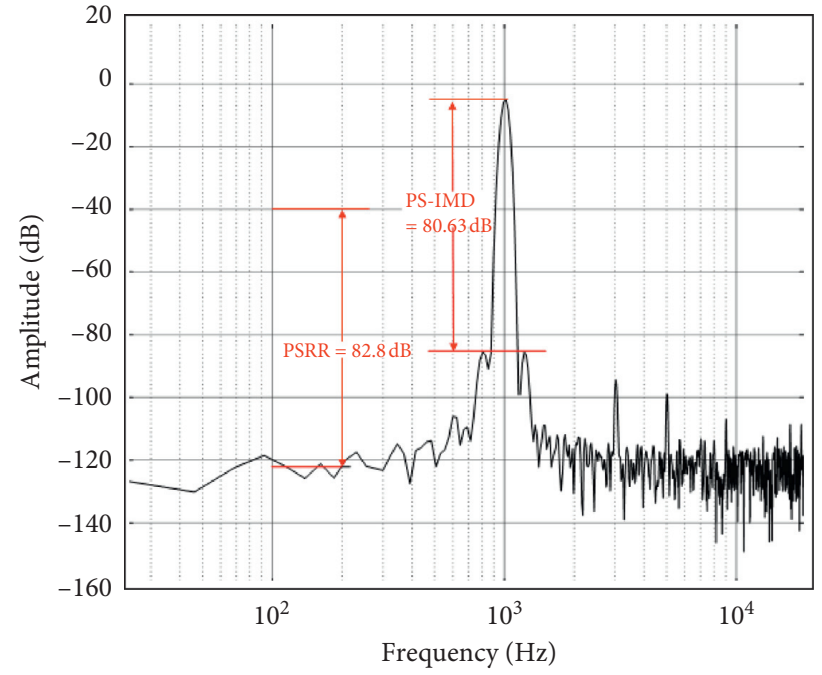

(b)

FIgURE 7: Continued. 


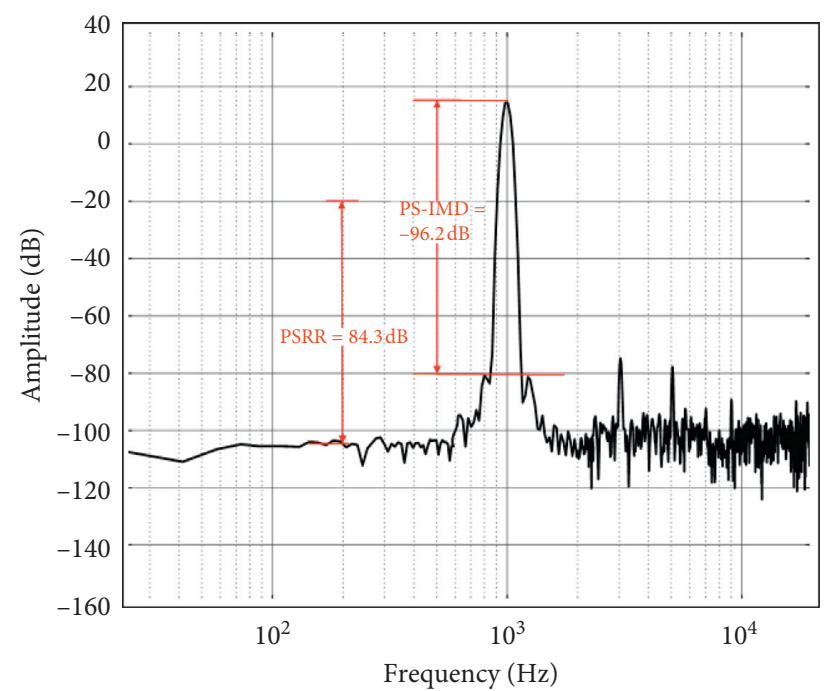

(c)

FIGURE 7: Spectrum diagram of power output signal. (a) Uncorrected. (b) FCLNF corrected. (c) FCLNF + FFPSNS corrected.

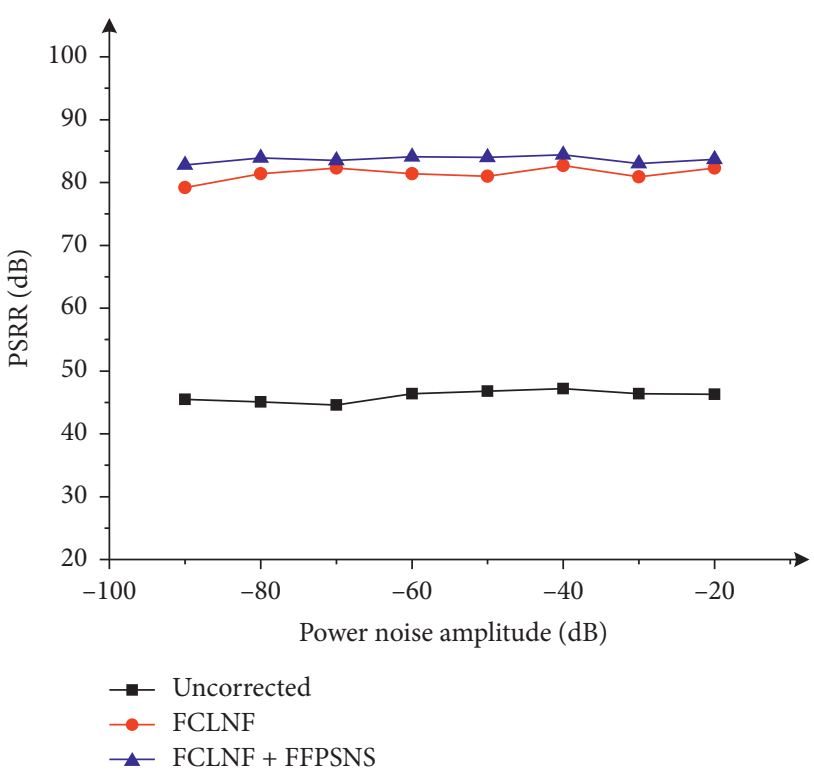

FiguRe 8: Contrast diagram of PSRR vs. power noise amplitude.

The power supply noise amplitude increased from $-90 \mathrm{~dB}$ to $-20 \mathrm{~dB}$. Regardless of whether it is calibrated or not, the power amplifier's PSRR remained basically unchanged with the increase of power supply noise amplitude, and the PSRR corrected by FCLNF and FCLNF + FFPSNS is significantly higher than that by uncorrected. The PSRR characteristics of the two correction schemes are basically the same.

The PS-IMD of the power amplifier does not change with the noise amplitude of the power supply. After FCLNF correction and FCLNF + FFPSNS correction, when the power supply noise amplitude is low $(<-70 \mathrm{~dB})$, PS-IMD is basically unchanged. This is because of the background noise in the output signal spectrum. The amplitude of the

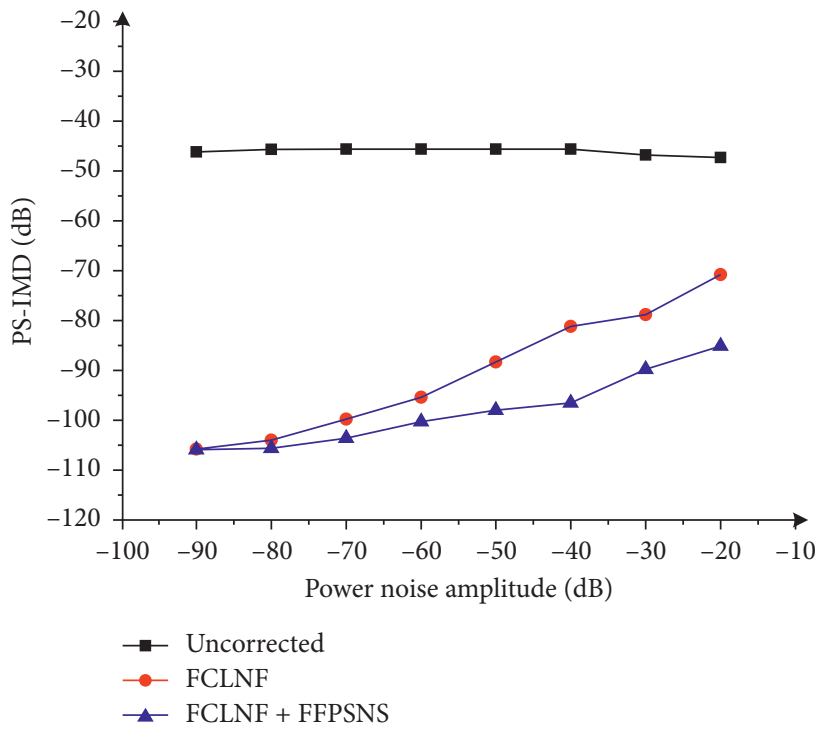

FIGURE 9: Contrast diagram of PS-IMD vs. power noise amplitude.

intermodulation components generated by the power supply noise and the input signal is basically the same as the background noise amplitude. However, as the noise amplitude of power supply continues to increase, PS-IMD of power amplifier starts to increase, and PS-IMD performance declines. This is due to the intermodulation component generated by the power supply noise and the input signal is higher than the background noise when the power supply noise amplitude increases to a certain extent and the PSIMD characteristics of the two correction schemes are basically equivalent.

Figure 10 compares the changes in the PSRR of the power amplifier with the power supply noise frequency when the three solutions are used. It can be seen from Figure 6 that, whether corrected or not, the PSRR of the 


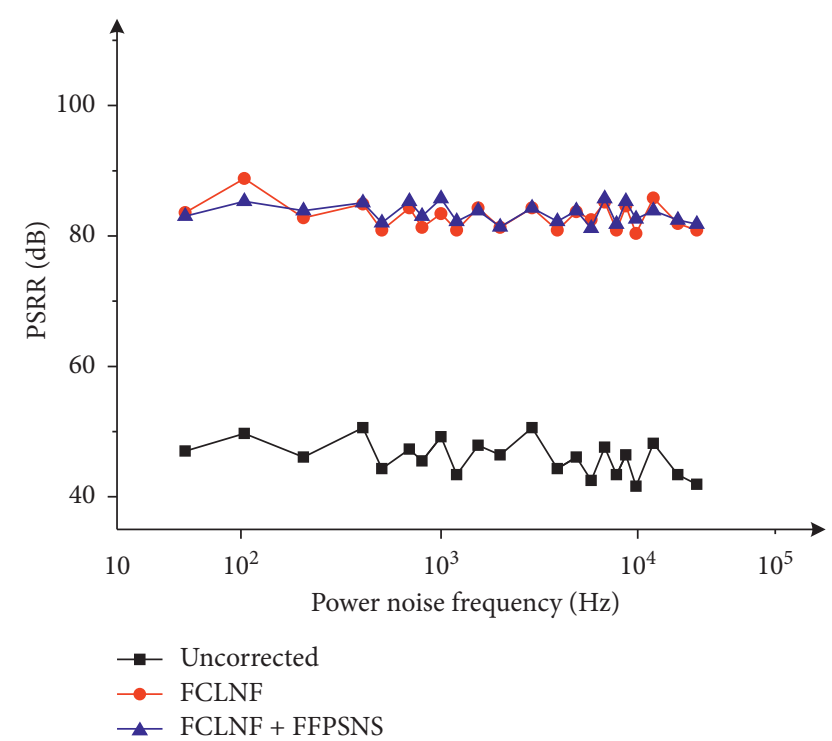

FIGURE 10: Contrast diagram of PSRR vs. power noise frequency.

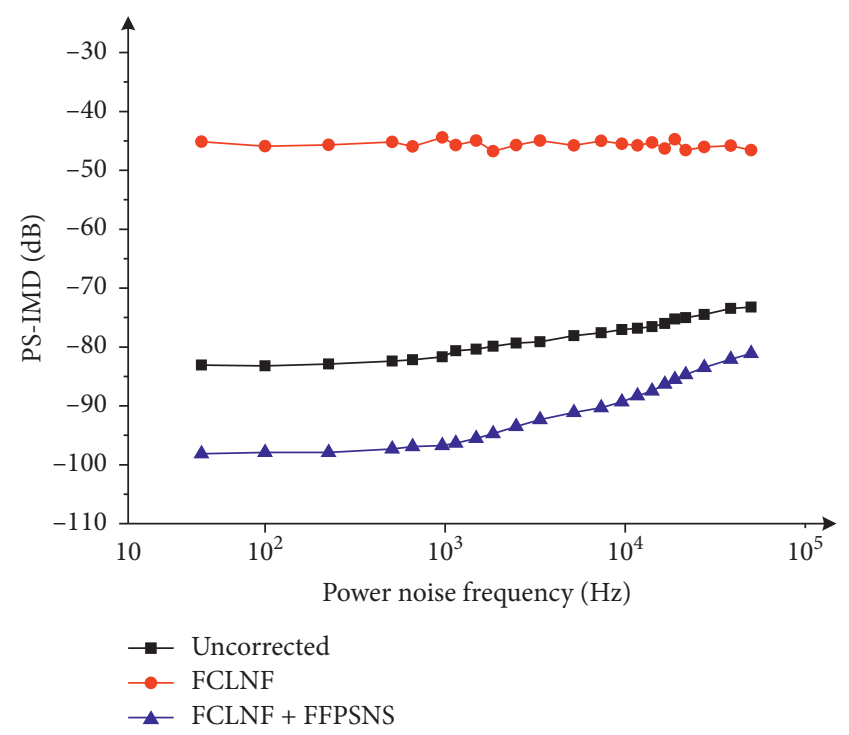

FIGURE 11: Contrast diagram of PS-IMD vs. power noise frequency.

power amplifier will not change drastically as the noise frequency of the power stage power supply increases, and the PSRR of the power amplifier after correction by LCLNF + FFPSNS and LCLNF is significantly higher than the PSRR of the uncorrected power amplifier.

Figure 11 compares the changes of the PS-IMD of the power amplifier with the power supply noise frequency when the three schemes are used. As the power supply noise frequency increases, the PS-IMD of the uncorrected power amplifier is relatively large and basically remains unchanged, while the PS-IMD of the power amplifier after correction using LCLNF and LCLNF + FFPSNS changes slowly at low frequencies $(<1000 \mathrm{~Hz})$. At high frequencies $(>1000 \mathrm{~Hz})$, the PS-IMD increases significantly, which is caused by the limited gain bandwidth product of the operational amplifier in the correction circuit, but the PS-IMD is smaller than the uncorrected one. At the same time, the PS-IMD of the power amplifier after LCLNF + FFPSNS correction is significantly smaller than the LCLNF correction scheme.

In summary, the PSRR of the power amplifier after correction by LCLNF and LCLNF + FFPSNS is basically not affected by the amplitude and frequency of the power stage power supply noise. With the change of the noise amplitude and frequency of the power stage power supply, the PS-IMD of the power amplifier after correction by LCLNF + FFPSNS is significantly lower than that after correction by LCLNF. At the same time, the power-stage power supply noise amplitude has a more significant impact on the PS-IMD of the power amplifier than the power supply noise frequency.

\section{Concluding Remarks}

Under the same experimental conditions, the two correction schemes were compared and analyzed. The results show that compared with the uncorrected power level, the performance of PSRR at $200 \mathrm{~Hz}$ source noise frequency is improved by $36.02 \mathrm{~dB}$ after FCLNF correction. The PS-IMD component was approximately reduced by $34.73 \mathrm{~dB}$. SNR performance improved by $27.11 \mathrm{~dB}$, and THD $+N$ decreased significantly. Compared with only using FCLNF error correction scheme, the performance of the power amplifier using FCLNF + FFPSNS error correction scheme is comparable at $200 \mathrm{~Hz}$ power supply noise frequency. However, the PS-IMD of the power amplifier decreased by $15.57 \mathrm{~dB}$. SNR was further improved by $17 \mathrm{~dB}$, and THD $+N$ dropped further down to $0.02 \%$.

\section{Data Availability}

The whole result data used to support the findings of this study are available from the corresponding author upon request.

\section{Conflicts of Interest}

The authors declare that they have no conflicts of interest.

\section{Acknowledgments}

This work was supported by Key Problems in Science and Technology of Henan Provincial (172102310671), Anyang Science and Technology Project and Anyang Institute of Technology Cultivation Project (YPY2019004), and Key Discipline Project of Henan Education Department (2018 [NO:119]).

\section{References}

[1] C. K. Lam, M. T. Tan, S. M. Cox, and K. S. Yeo, "Class-D amplifier power stage with PWM feedback loop," IEEE Transactions on Power Electronics, vol. 28, no. 8, pp. 38703881, 2013.

[2] Z. Yu, F. Wang, and Y. Fan, "A power supply error correction method for single-ended digital audio class D amplifiers," International Journal of Electronics, vol. 103, no. 12, pp. 2110-2124, 2016. 
[3] Z. Zhou, Y. Shi, X. Ming, B. Zhang, Z. Li, and Z. Chen, "Modelling and analysis of a high-performance class $\mathrm{D}$ audio amplifier using unipolar pulse-width-modulation," International Journal of Electronics, vol. 99, no. 2, pp. 163-177, 2012.

[4] H. Zheng, Z. Zhu, and R. Ma, "A. 0.02\% THD and $80 \mathrm{~dB}$ PSRR filterless class $\mathrm{D}$ amplifier with direct lithium battery hookup in mobile application," Journal of Semiconductors, vol. 38, no. 7, pp. 56-63, 2017.

[5] H. He, Y. Kang, and J. Yu, "A novel low-power high-efficiency 3-state filterless bang-bang class D amplifier," in Proceedings of IEEE International Conference On Electronics, Cairo, Egypt, December 2015.

[6] H. Zheng, Q. Wang, and Y. Liu, "A low THD + N and high PSRR class-D power amplifier in mobile application," Guti Dianzixue Yanjiu Yu Jinzhan/Research and Progress of Solid State Electronics, vol. 37, no. 2, pp. 134-139, 2017.

[7] A. Donida, P. Malcovatip, A. Nagari, R. Cellier, and A. Baschirotto, "A 40-nm CMOS, 1.1-V, 101-dB DR, 1.7-mW continuous-time $\Sigma \Delta$ ADC for a digital closed-loop class-D amplifier," in Proceedings of 2013 IEEE 20th International Conference On Electronics, Circuits, and Systems (ICECS), Abu Dhabi, UAE, December 2013.

[8] F. Mostert and D. Schinkel, W. Groothedde, A $5 \times 80 \mathrm{~W} 0$. $004 \%$ THD $+\mathrm{N}$ automotive multiphase class-D audio amplifier with integrated low-latency $\Delta \Sigma$ ADCs for digitized feedback after the output filter," in Proceedings of IEEE International Solid-State Circuits C.Solid-state Circuits Conference, San Francisco, CA, USA, February 2017.

[9] X. Chen, H. Qu, Z. Yu, C. Zhang, and E. Zhang, “A filterless digital audio class-D amplifier based on grow-left double-edge pulse width modulation," in Proceedings of 2nd International Conference on Integrated Circuits and Microsystems, Nanjing, China, November 2017.

[10] R. Cellier, G. Pillonnet, and N. Abouchi, "Analysis and design of an analog control loop for digital input class D amplifiers," in Proceedings of IEEE 18th International Conference on Electronic,Circuits and Systems, Beirut, Lebanon, December 2011.

[11] D. Lee, J. Noh, and J. Lee, "A class-D amplifier for a digital hearing aid with $0.015 \%$ total harmonic distortion plus noise," ETRI Journal, vol. 35, no. 5, pp. 819-826, 2013.

[12] J. Noh, D. Lee, J.-G. Jo, and C. Yoo, “A class-D amplifier with pulse code modulated (PCM) digital input for digital hearing aid," IEEE Journal of Solid-State Circuits, vol. 48, no. 2, pp. 465-472, 2013.

[13] K. Philips and J. Van Den, "Power DAC: a single-chip audio DAC with a $70 \%$-efficient power stage in $0.5 \mu \mathrm{m}$ CMOS," in Proceedings of Digest Of Technical Papers Of the 1999 IEEE International Solid-State Circuits Conference, San Francisco, CA, USA, February 1999.

[14] S. Ramaswamy, J. Krishnan, B. Forejt, J. Joy, M. Burns, and G. Burra, "A high-performance digital-input class-D amplifier with direct battery connection in a $90 \mathrm{~nm}$ digital CMOS process," in Proceedings of Digest Of Technical Papers Of the 2008 IEEE International Solid-State Circuits Conference, San Francisco, CA, USA, February 2008.

[15] S.-H. Chien, Y.-W. Chen, and T.-H. Kuo, "A low quiescent current, low THD $+\mathrm{N}$ class-D audio amplifier with area-efficient PWM-residual-aliasing reduction," IEEE Journal of Solid-State Circuits, vol. 53, no. 12, pp. 3377-3385, 2018.

[16] C. W. Lin and B. S. Hsieh, "Dynamic power efficiency improvement for PWM class-D amplifier," IEICE Electronics Express, vol. 10, no. 6, 2013.
[17] A. I. Colli-Menchi, J. Torres, and E. Sanchez-Sinencio, "A feed-forward power-supply noise cancellation technique for single-ended class-D audio amplifiers," IEEE Journal of SolidState Circuits, vol. 49, no. 3, pp. 718-728, 2014.

[18] V. Adrian, K. Cui, B. H. Geww, and J. S. Chang, "A Randomized Modulation scheme for filterless digital class D audio amplifiers," in Proceedings of IEEE International Symposium on Circuits and Systems, Melbourne VIC, Australia, June 2014.

[19] F. Guanzurolif, R. Bassoli, C. Crippa, D. Devecchi, and G. Nicollini, "A 1 W 104 dB SNR filter-less fully-digital openloop class D audio amplifier with EMI reduction," IEEE Journal of Solid-State Circuits, vol. 47, no. 3, pp. 686-698, 2012. 